\section{OPEN ACCESS}

Edited by:

LiZUo,

Ohio State University, USA

Reviewed by:

Martina Krüger,

University of Düsseldorf, Germany

Han-Zhong Feng,

Wayne State University School of

Medicine, USA

Zewen Liu,

Ohio State University, USA

*Correspondence:

Bernard Geny

bernard.geny@chru-strasbourg.fr

${ }^{\dagger}$ These authors have contributed equally to this work.

Specialty section:

This article was submitted to

Striated Muscle Physiology,

a section of the journal

Frontiers in Physiology

Received: 06 October 2016 Accepted: 19 January 2017

Published: 06 February 2017

Citation:

Charles A-L, Guilbert A-S, Guillot M, Talha S, Lejay A, Meyer A, Kindo M, Wolff V, Bouitbir J, Zoll J and Geny B

(2017) Muscles Susceptibility to

Ischemia-Reperfusion Injuries

Depends on Fiber Type Specific

Antioxidant Level. Front. Physiol. 8:52.

doi: 10.3389/fphys.2017.00052

\title{
Muscles Susceptibility to Ischemia-Reperfusion Injuries Depends on Fiber Type Specific Antioxidant Level
}

\begin{abstract}
Anne-Laure Charles ${ }^{1,2 \dagger}$, Anne-Sophie Guilbert ${ }^{1,3+}$, Max Guillot ${ }^{1,4}$, Samy Talha ${ }^{1,2}$, Anne Lejay ${ }^{1,2}$, Alain Meyer ${ }^{1,2}$, Michel Kindo ${ }^{1,5}$, Valérie Wolff ${ }^{1,6}$, Jamal Bouitbir ${ }^{7}$, Joffrey Zoll ${ }^{1,2}$ and Bernard Geny ${ }^{1,2 *}$
\end{abstract}

1 Equipe d'accueil 3072, Faculté de Médecine, Fédération de Médecine Translationnelle, Université de Strasbourg, Strasbourg, France, ${ }^{2}$ Service de Physiologie et d'Explorations Fonctionnelles, Pôle de Pathologie Thoracique, Nouvel Hôpital Civil, CHRU de Strasbourg, Strasbourg, France, ${ }^{3}$ Service de Réanimation Médico-Chirurgicale Pédiatrique Spécialisée, Hôpital de Hautepierre, CHRU de Strasbourg, Strasbourg, France, ${ }^{4}$ Service de Réanimation Médicale, Hôpital de Hautepierre, CHRU de Strasbourg, Strasbourg, France, ${ }^{5}$ Service de Chirurgie Cardio-Vasculaire, Pôle d'activité Médico-chirurgicale Cardiovasculaire, Nouvel Hôpital Civil, CHRU de Strasbourg, Strasbourg, France, ${ }^{6}$ Unité neurovasculaire, Hôpital de Hautepierre, CHRU de Strasbourg, Strasbourg, France, ${ }^{7}$ Division of Clinical Pharmacology and Toxicology, University Hospital Basel, Basel, Switzerland

Muscle injury resulting from ischemia-reperfusion largely aggravates patient prognosis but whether and how muscle phenotype modulates ischemia-reperfusion-induced mitochondrial dysfunction remains to be investigated. We challenged the hypothesis that glycolytic muscles are more prone to ischemia-reperfusion-induced injury than oxidative skeletal muscles. We therefore determined simultaneously the effect of $3 \mathrm{~h}$ of ischemia induced by aortic clamping followed by $2 \mathrm{~h}$ of reperfusion $(\mathrm{IR}, n=11)$ on both gastrocnemius and soleus muscles, as compared to control animals $(C, n=11)$. Further, we investigated whether tempol, an antioxidant mimicking superoxide dismutase, might compensate a reduced defense system, likely characterizing glycolytic muscles (IR-Tempol, $n=7$ ). In the glycolytic gastrocnemius muscle, as compared to control, ischemia-reperfusion significantly decreased mitochondrial respiration $(-30.28 \pm 6.16 \%$, $p=0.003)$, increased reactive oxygen species production $(+79.15 \pm 28.72 \%, p=0.04)$, and decreased reduced glutathione $(-28.19 \pm 6.80 \%, p=0.011)$. Less deleterious effects were observed in the oxidative soleus muscle $(-6.44 \pm 6.30 \%,+4.32 \pm 16.84 \%$, and $-8.07 \pm 10.84 \%$, respectively), characterized by enhanced antioxidant defenses (0.63 \pm 0.05 in gastrocnemius vs. $1.24 \pm 0.08 \mu \mathrm{mol} \mathrm{L}^{-1} \mathrm{~g}^{-1}$ in soleus). Further, when previously treated with tempol, glycolytic muscle was largely protected against the deleterious effects of ischemia-reperfusion. Thus, oxidative skeletal muscles are more protected than glycolytic ones against ischemia-reperfusion, thanks to their antioxidant pool. Such pivotal data support that susceptibility to ischemia-reperfusion-induced injury differs between organs, depending on their metabolic phenotypes. This suggests a need to adapt therapeutic strategies to the specific antioxidant power of the target organ to be protected.

Keywords: antioxidant, ischemia-reperfusion, metabolic phenotype, mitochondria, muscle, sarcopenia, oxidative stress, peripheral arterial disease (PAD) 


\section{INTRODUCTION}

Lower limb ischemia is a frequent clinical problem resulting from trauma, hemorrhage, vascular occlusion, and/or thromboembolic events (Blaisdell, 2002; Ali et al., 2007; Beekley et al., 2008). Surgical or medical reperfusion is needed to avoid definitive necrosis, and previous studies showed that muscle irreversible damages appear likely after $4 \mathrm{~h}$ of ischemia (Belkin et al., 1988; Carvalho et al., 1997; Blaisdell, 2002; Martou et al., 2006; Paradis et al., 2016). After 3 h of ischemia, injury has shown to be reversible following 28 days of reperfusion. In this case however, reversibility was likely afforded by muscle regeneration (Vignaud et al., 2010; Itoh et al., 2013).

Albeit necessary, reintroduction of oxygenated blood into the ischemic areas induces unfortunately ischemia-reperfusion (IR) injury. This is significant, aggravating both local and general prognosis of the patient (Harris et al., 1986; Blaisdell, 2002; Martou et al., 2006) and supporting continuous work in order to reduce these deleterious effects.

Recently major advances have been observed in our knowledge of lower limb IR pathophysiology. Notably, interactions between muscle mitochondria and reactive oxygen species (ROS) have been emphasized (Lejay et al., 2014). Oxidative stress, which precedes mitochondrial dysfunction, arises during ischemia and is enhanced after reperfusion (Guillot et al., 2014). This suggests that modulating ROS production may reduce IR-induced injury. Accordingly, we and others demonstrated that muscle mitochondrial function is protected when ROS production is reduced and/or when the ROS produced can be efficiently handled by the antioxidant system. Such therapeutic approaches were based on both ischemic and pharmacologic conditioning (Ali et al., 2007; Makris et al., 2007; Charles et al., 2011; Tran et al., 2011; Talha et al., 2013).

Interestingly, however, interactions between mitochondria and ROS are more subtle. Significant increases in ROS production are generally considered deleterious because it induces lipid peroxidation, protein carbonylation, and mitochondrial dysfunction that is associated with reduced ATP production (Anderson and Neufer, 2006; Magder, 2006). On the other hand, a small ROS increase is a signaling pathway stimulating mitochondrial biogenesis and enhancing antioxidant defenses (Ristow, 2014; Yun and Finkel, 2014). Recently, we reported that skeletal muscles are more prone to statin-induced injury than cardiac muscles (Bouitbir et al., 2011). Metabolic phenotypes greatly differ between cardiac and skeletal muscles. Indeed, heart is highly oxidative, in association with enhanced mitochondrial respiration and antioxidant system (Masuda et al., 2003; Kowaltowski et al., 2009; Bouitbir et al., 2011).

Similar differences exist among skeletal muscles, which are classified mainly depending on their contractile and/or metabolic phenotype. Mammalian skeletal muscles are characterized by their abilities to adapt to new conditions. Indeed, changes can be detected by multiple sensors, from membrane receptors for hormones and cytokines, to metabolic sensors that detect variations in the high-energy phosphate concentration, oxygen as well as oxygen free radicals. These sensors trigger cascades of signaling pathways which may ultimately lead to changes in fiber type, especially at the level of metabolic properties (for review see
Pourova et al., 2010; Schiaffino and Reggiani, 2011; Blaauw et al., 2013; Zuo et al., 2015).

Particularly, certain fibers possess a large amount of mitochondria, and rely mainly on oxidative phosphorylation. These fibers can be defined as "oxidative:" they require more oxygen and depend hence on the vascularisation to synthesize ATP. On the other hand, other fibers possess few mitochondria, and mainly rely on glycolysis to produce ATP. These fibers can be identified as "glycolytic" (Jackman and Willis, 1996; Meyer et al., 2014). Moreover, the analysis of the expression myosin heavy chains enables to differentiate four subtypes of slow (Type I) and fast twitch fibers: IIA, IIX, and IIB (Talbot and Maves, 2016). Type I fiber, such as the soleus, is considered as a slow oxidative fiber with high baseline respiratory rates and important antioxidant pools. Type IIA fibers use both oxidative and glycolytic metabolisms to produce ATP, and have a relatively high mitochondrial content (however lower than type I fibers). These fibers are relatively resistant to fatigue (Schiaffino, 2010). Type IIB fibers possess a low amount of mitochondria and rely mainly on glycolysis. These fibers has less antioxidant enzymes compared with type I fibers (Powers and Hamilton, 1999; Ploquin et al., 2012) and are easily fatigable, but develop tremendous amounts of strength. Type IIX fibers possess an intermediate phenotype between type IIA and type IIB fibers. Based on these characteristics, and on the fact that superficial gastrocnemius present with $97 \%$ of type IIb fibers and that soleus present with $30.6 \%$ of type I fibers (Bloemberg and Quadrilatero, 2012), we decided to study both superficial gastrocnemius and soleus in this study.

Interestingly, although Turoczi et al. described a type I muscle altered viability (Turóczi et al., 2014), other studies rather supported glycolytic muscles alterations after IR. Particularly, considering contractile force, Demirel et al. observed differences between glycolytic and oxidative muscles submitted to IR. After $4 \mathrm{~h}$ ischemia and $2 \mathrm{~h}$ reperfusion, the force-frequency curve was more altered in extensor digitorum longus (EDL) than in soleus. Further, resistance to fatigue was lower in EDL than in soleus (Demirel et al., 2013). Impaired fatigue resistance and contraction were also observed after IR in gastrocnemius (Zhang et al., 2017) and accordingly, proteins implicated in muscle contraction are known to be targets for ROS (Beckendorf and Linke, 2015), which are largely implicated in IR pathophysiology (increased lipid peroxidation and oxidized proteins; Avci et al., 2012).

Considering that oxidative skeletal muscles can also suffer from intense IR (Ali et al., 2010; Balogh et al., 2014; Turóczi et al., 2014; He and Zuo, 2015), we aimed to challenge the hypothesis their susceptibility to IR injuries might depend on their metabolic phenotypes. Particularly, oxidative skeletal muscles (as compared to glycolytic ones) should be protected from IR injuries, thanks to their antioxidant defenses.

\section{MATERIALS AND METHODS}

\section{Ethical Approval}

Procedures were conducted in accordance with US National Institutes of Health guidelines. The study was approved by the institutional animal care committee of the University 
of Strasbourg (Comité Régional d'Ethique en Matière d'Expérimentation Animale de Strasbourg, CREMEAS, CEE35) (AL/02/09/04/08).

\section{Animals}

We worked on adult male Wistar rats (Depré, Saint-Doulchard, France) aged 8 weeks. The temperature environment is regulated at $22 \pm 2^{\circ} \mathrm{C}$, and the room is submitted to a 12-h light-dark cycle. Animals had free access to food and water.

\section{Preoperative Management}

The anesthesia was induced in a hermetic cage with $4 \%$ isoflurane (Aerrane, CSP, Cournon, France) and oxygen. During the operation, the animals ventilated spontaneously, with an oxygen-delivering mask, and adapted isoflurane concentrations. The body temperature was controlled and maintained (Homeothermic blanket control unit, MINERVE, Harvard Apparatus ${ }^{\circledR}$, Esternay, France).

\section{Surgical Procedures and Experimental Design}

Twenty nine rats were divided in three groups (Figure 1): the control group (C, $n=11)$ underwent 5 h of general anesthesia. A midline laparotomy was performed and the abdominal aorta was exposed like in the IR groups.

The ischemia-reperfusion (IR) group $(n=11)$ underwent $3 \mathrm{~h}$ of ischemia induced by infra-renal aortic occlusion, and collateral vessel coagulation $\left(\right.$ GEIGER $^{\circledR}$, thermal cautery unit, Geiger Medical Technologies, Council Bluffs, Iowa, USA) followed by 2 $\mathrm{h}$ of reperfusion. As previously reported, ischemia was clinically characterized by cyanosis and lack of arterial pulse distal to the clamp and reperfusion was associated with recoloring and pulse return in the hind limb (Thaveau et al., 2007; Mansour et al., 2011; Pottecher et al., 2013).

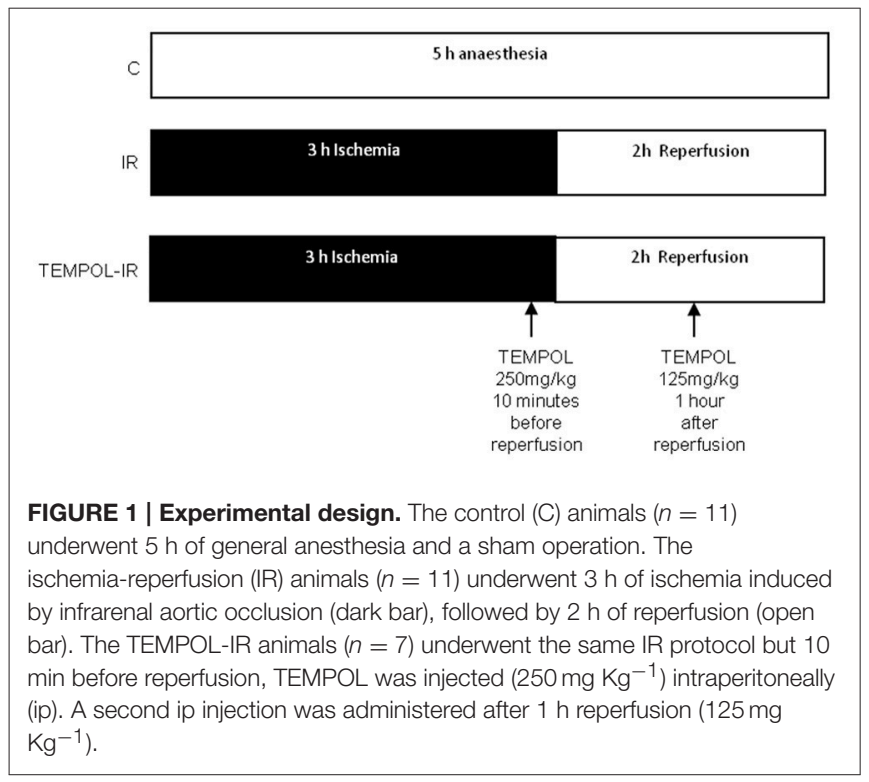

Duration of ischemia was based on our and previous data demonstrating that $3 \mathrm{~h}$ ischemia is needed to result in significant skeletal muscle injury, which is still more severe after $6 \mathrm{~h}$ ischemia (Belkin et al., 1988; Thaveau et al., 2007). Chronological sequences about the cellular and molecular events occurring during skeletal muscle IR has been recently reported (Paradis et al., 2016). Interestingly, as compared to the heart which is still beating, such a long duration might be explained by the noncontracting, resting skeletal muscle generally examined. Like in the heart however (Ma et al., 2016), a no reflow occurrence might increase with long ischemia time and we therefore choose a $3 \mathrm{~h}$ ischemia period for our experimental protocol.

At the end of reperfusion, lactate, which is considered as a good marker of anaerobic state, was determined on total blood obtained from the tail before and after ischemia (lactate Pro device, LT710, Arkray ${ }^{\circledR}$, KGK, Kyoto, Japan). Its decrease after opening the artery supports a successful reperfusion (Noll et al., 2012).

At the end of experiment, oxidative soleus and glycolytic superficial gastrocnemius muscles were excised (Bloemberg and Quadrilatero, 2012). For each muscle, one part was used immediately for oxidative capacities and ROS determinations. The second part was frozen in isopentane cooled by liquid nitrogen or directly in liquid nitrogen and thereafter stored for a later use.

Finally, to identify the role of oxidative stress in IR-related injury, and particularly to determine the potential protective effect of an anti-oxidant in gastrocnemius, a third group was given the superoxide dismutase mimetic. Rats from the Tempol-IR group $(n=7)$ had two intraperitoneal injections of 4-hydroxy2,2,6,6-tetramethylpiperidine-1-oxyl (Tempol, Sigma-Aldrich ${ }^{\circledR}$, St. Louis, MO, USA); $250 \mathrm{mg} \mathrm{kg}^{-1}$ at $10 \mathrm{~min}$ before the beginning of reperfusion and $125 \mathrm{mg} \mathrm{kg}^{-1}$ after $1 \mathrm{~h}$ of reperfusion (Halter et al., 2010).

\section{Study of Muscle Mitochondrial Respiration}

All used products were purchased from Sigma-Aldrich ${ }^{\circledR}$ (St. Louis, MO, USA).

We studied the mitochondrial respiration of skinned fibers rather than isolated mitochondria to preserve the environment and the integrity of mitochondria (Saks et al., 1998; Rasmussen and Rasmussen, 2000). The two muscles studied were superficial gastrocnemius and soleus muscles.

Oxygen consumption was measured by using a Clarktype electrode in an oxygraphic cell (Strathkelvin Instruments, Glasgow, Scotland) as previously described (Kuznetsov et al., 2008; Charles et al., 2011; Kindo et al., 2012, 2016). Maximal oxidative respiration $\left(\mathrm{V}_{\max }\right)$ was measured after adding ADP $\left(2 \mathrm{mmol} \mathrm{L}{ }^{-1}\right)$ as phosphate acceptor and glutamate $(5 \mathrm{mmol}$ $\left.\mathrm{L}^{-1}\right)$-malate $\left(2 \mathrm{mmol} \mathrm{L}{ }^{-1}\right)$ as mitochondrial substrates. $\mathrm{V}_{\max }$ reflects the electron flow through complexes I, III, IV, and ATP synthase.

Then, complex I was blocked with amobarbital $(0.02 \mathrm{mmol}$ $\left.\mathrm{L}^{-1}\right)$, and complex II was subsequently stimulated $\left(\mathrm{V}_{\text {succ }}\right)$ with succinate $\left(25 \mu \mathrm{mol} \mathrm{L}{ }^{-1}\right)$. The electron flow went through complexes II, III, IV, and ATP synthase. 
Finally, $\quad \mathrm{N}, \mathrm{N}, \mathrm{N}^{\prime}, \mathrm{N}^{\prime}$-tetra-methyl-p-phenylenediamine

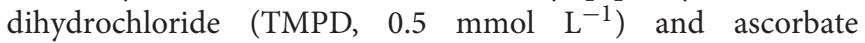
(0.5 mmol $\left.\mathrm{L}^{-1}\right)$ were added as artificial electron donors to cytochrome c, to study complex IV activity. After the experiment, fibers were harvested and dried for $15 \mathrm{~min}$ at $150^{\circ} \mathrm{C}$, and respiration rates were expressed as $\mu \mathrm{mol} \mathrm{O}_{2} \mathrm{~min}^{-1} \mathrm{~g}^{-1} \mathrm{dry}$ weight.

\section{Electron Paramagnetic Resonance}

Although one method might have been sufficient, we decided to perform two complementary methods for ROS measurement in order to comfort the data. DHE staining is very useful and allows demonstrate the ROS level in tissue and EPR is viewed as an excellent technique, measuring the ROS production by the tissue (Dikalov et al., 2007; Kuznetsov et al., 2011; Lejay et al., 2015).

Electron Paramagnetic Resonance (EPR) allows determining superoxide $\left(\mathrm{O}_{2}^{-}\right)$formation.

$\mathrm{O}_{2}^{--}$concentration was determined with a specific spin probe 1-hydroxy-3-methoxycarbonyl-2, 2, 5, 5-tetramethylpyrrolidine hydrochloride (CMH, Noxygen ${ }^{\circledR}$, Elsach, Germany), oxidized by impaired electron (Dikalov et al., 2007; Lejay et al., 2015; Kindo et al., 2016).

All used substrates for the Krebs Hepes buffer were purchased from Sigma-Aldrich ${ }^{\circledR}$ (St. Louis, MO, USA).

Ten $2-\mathrm{mm}$ pieces of muscles were cut and washed twice with the Krebs Hepes Buffer $\left(\mathrm{NaCl} 99 \mathrm{mmol} \mathrm{L}^{-1}\right.$; $\mathrm{KCl} 4.69 \mathrm{mmol}$

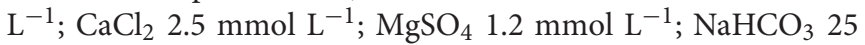
mmol L ${ }^{-1} ; \mathrm{KH}_{2} \mathrm{PO}_{4} 1.03 \mathrm{mmol} \mathrm{L}{ }^{-1} ; \mathrm{D}(+)$ Glucose $5.6 \mathrm{mmol}$ $\mathrm{L}^{-1}$; Na-hepes $20 \mathrm{mmol} \mathrm{L}^{-1}$; $\left.\mathrm{pH} 7.4\right)$ containing $25 \mu \mathrm{mol} \mathrm{L} \mathrm{L}^{-1}$ deferoxamine and $5 \mu \mathrm{mol} \mathrm{L}{ }^{-1}$ diethyldithiocarbamate (DETC) to minimize $\mathrm{CMH}$ auto-oxidation. Then, they were incubated in a plate at $37^{\circ} \mathrm{C}$ with the spin probe $\mathrm{CMH}(200 \mu \mathrm{mol}$ $\left.\mathrm{L}^{-1}\right)$ for $30 \mathrm{~min}$ under controlled pressure $(20 \mathrm{mmHg})$ and gas mix $\left(\mathrm{N}_{2}: 97.8 \%, \mathrm{O}_{2}: 2.8 \%\right)$ to mimic tissular environment (Noxygen ${ }^{\circledR}$, Elsach, Germany). The incubation of tissues is stopped by placing the plate on ice. Forty-one microliters of the supernatant are injected in a disposable capillary tube, and placed inside the cavity of the e-scan spectrometer (Bruker Win-EPR ${ }^{\circledR}$, Elsach, Germany) for data acquisition at $15^{\circ} \mathrm{C}$ (The used EPR settings: Centre Field $3461.144 \mathrm{~g}$, microwave power $21.85 \mathrm{~mW}$, modulation amplitude $2.40 \mathrm{G}$, sweep time $5.24 \mathrm{~s}$ (10 scans), sweep width $60 \mathrm{G}$, number of lag curve points 1 ).

The amplitude of the signal is measured, and the concentration of CM-radical is determined by calibration with standard concentrations of the radicals CM. After the EPR measurement, pieces of muscles are dried for $15 \mathrm{~min}$ at $150^{\circ} \mathrm{C}$,

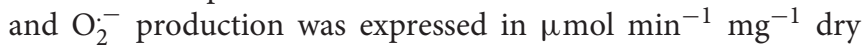
weight.

\section{Dihydroethidium Staining}

Dihydroethidium staining (DHE, Sigma-Aldrich ${ }^{\circledR}$ ) allows detecting the presence of ROS in skeletal muscles.

Frozen muscles were cut on serial sections (10 $\mu$ m-thicks) with a cryostat microtome and thaw mounted on glass slides and incubated with DHE $\left(2.5 \mu \mathrm{mol} \mathrm{L} \mathrm{L}^{-1}\right)$. DHE produced red fluorescence when oxidized to ethidium bromide (EtBr) by $\mathrm{O}_{2}^{-}$ (Mulsch et al., 2001; Talha et al., 2013; Guillot et al., 2014). As compared to other markers, the interest of DHE is its relatively good specificity to $\mathrm{O}_{2}^{-}$.

After staining, sections were examined under an epifluorescence microscope (Eclipse E800, Nikon, New York, USA) with a $20 \mathrm{X}$ epifluorescence objective. The emission signal was recorded with a Zeiss 573-637 nm filter. We analyzed micrographs with Adobe Photoshop 6.0 (USA). The results were reported in percentage of control group.

\section{Measurement of Reduced Glutathione}

The glutathione peroxidase is an important intracellular antioxidant, because it is the primary enzyme responsible for $\mathrm{H}_{2} \mathrm{O}_{2}$ detoxification in mitochondria (Antunes et al., 2002; Zoccarato et al., 2004).

All used products were purchased from Sigma-Aldrich ${ }^{\circledR}$ (St. Louis, MO, USA).

Reduced glutathione (GSH) was measured in the different muscles by monitoring the reduction of 5,5'-dithio-bis-(2nitrobenzoic acid) (DTNB) to 5-thio-2-nitrobenzoate (TNB) in presence of GSH at $412 \mathrm{~nm}$ (Akerboom and Sies, 1981; Bouitbir et al., 2011). Briefly, to determine GSH content, tissue samples were mixed with cold 5\% 5-sulfosalicylic acid, after $10 \mathrm{~min}$ of incubation on ice, the mixture was centrifuged at $4000 \mathrm{rpm}$ for $10 \mathrm{~min}$ at $4^{\circ} \mathrm{C}$ to remove proteins. The samples were then diluted in cold MOPS buffer, at room temperature. The diluted sample was added to glutathione reductase (24 units $\mathrm{mL}^{-1}$ ) and MOPS $\left(0.1 \mathrm{~mol} \mathrm{~L}^{-1}\right)$ containing $1 \mathrm{mmol} \mathrm{L}^{-1}$ EDTA, 0.3 mmol L ${ }^{-1} \mathrm{NADPH}$, and $0.22 \mathrm{mmol} \mathrm{L}^{-1} \mathrm{DTNB}$. The absorbance was continuously monitored for $1 \mathrm{~min}$, as an index of DTNB reduction by GSH contained in the sample. Reduced glutathione was expressed in $\mu \mathrm{mol} \mathrm{L} \mathrm{L}^{-1} \mathrm{~g}^{-1}$.

\section{Statistical Analysis}

Values are expressed as mean \pm SEM. $n$ indicates the number of animals. Statistical analyses were performed with Student's $t$-test for unpaired data. When the variances were significantly different, we used the Student's unpaired $t$-test with Welch's correction. For the lactate rates comparison statistical significance was measured by ANOVA followed by the post-test Newman Keuls (GraphPad Prism 5, Graph Pad Software, Inc., San Diego, CA, USA). Values of $p<0.05$ were considered to be statistically significant.

\section{RESULTS}

\section{Ischemia Significantly Increased Blood Lactates, Which Returned to Baseline Values after Reperfusion (Figure 2)}

Plasma lactate kinetic demonstrated the efficiency of IR. After 3 $\mathrm{h}$ ischemia, lactate increased significantly (10.9 \pm 2.0 vs. $2.2 \pm$ $\left.0.2 \mathrm{mmol} \mathrm{L}^{-1},+395.4 \% ; p<0.001\right)$. Then, at reperfusion, lactate decreased significantly $\left(3.1 \pm 0.5\right.$ vs. $10.9 \pm 2.0 \mathrm{mmol} \mathrm{L}^{-1}, p<$ 0.001 ) and there was no significant difference in lactate values between before ischemia and after reperfusion $(2.2 \pm 0.2$ vs. 3.1 $\pm 0.5 \mathrm{mmol} \mathrm{L}^{-1}$, NS, respectively). 


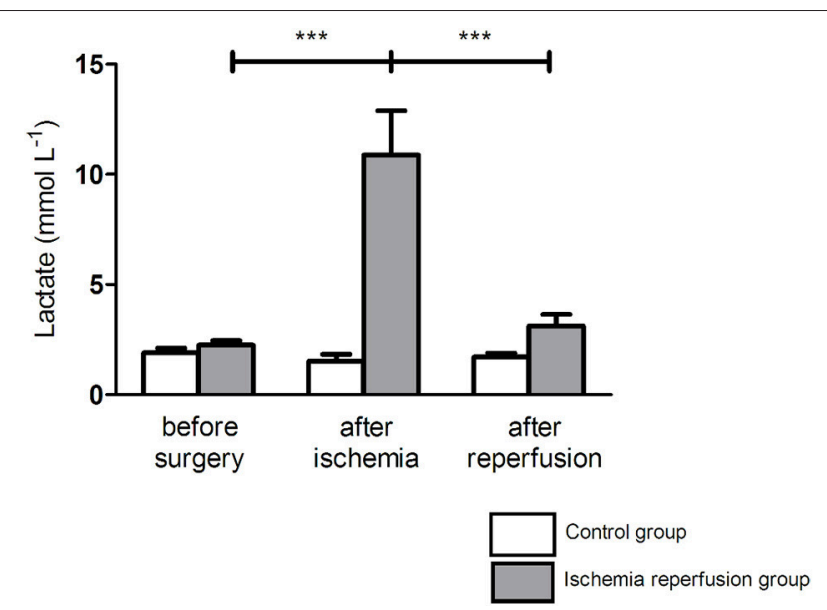

FIGURE 2 | Lactate concentrations increased with ischemia and returned to baseline level after reperfusion. Ischemia-reperfusion (IR) group (gray bars), control group (white bars). Lactates levels were measured before and after $3 \mathrm{~h}$ of ischemia, and after $2 \mathrm{~h}$ of reperfusion. Results were expressed as mean \pm SEM. ${ }^{\star * *} p<0.001$ compared to after ischemia.

\section{IR Impaired Mitochondrial Respiration, Increased ROS Production, and Reduced Antioxidant Defenses in Glycolytic Gastrocnemius Muscles (Figure 3) Mitochondrial Respiratory Chain Complex Activities (Figure 3A)}

In gastrocnemius, maximal respiration rate $\left(\mathrm{V}_{\max }\right)$, reflecting the activity of complexes I, III, IV, and V, was significantly altered after IR as compared to the control group $(4.51 \pm 0.44 v s .6 .61 \pm$ $0.42 \mu \mathrm{mol} \mathrm{O}_{2} \mathrm{~min}^{-1} \mathrm{~g}^{-1}$ dry weight, respectively, $p<0.05$ ).

Similarly, $\mathrm{V}_{\text {succ }}$, reflecting the activity of complexes II, III, IV, and $V$, was decreased after IR $(3.82 \pm 0.36$ vs. $6.23 \pm 0.61 \mu \mathrm{mol}$ $\mathrm{O}_{2} \min ^{-1} \mathrm{~g}^{-1}$ dry weight, respectively, $p<0.01$ ).

Finally, $\mathrm{V}_{\mathrm{TMPD}}$ /Asc, reflecting the complex IV activity, was decreased after IR $\left(14.05 \pm 1.92\right.$ vs. $20.61 \pm 1.49 \mu \mathrm{mol} \mathrm{O}_{2} \mathrm{~min}^{-1}$ $\mathrm{g}^{-1}$ dry weight compared to the control group, respectively, $p<$ $0.05)$.

Thus, IR impaired all complexes of the mitochondrial respiratory chain of the gastrocnemius muscle.

\section{ROS Production (Figures 3B,C)}

The graph $\mathrm{B}$ represents the $\mathrm{O}_{2}^{-}$production measured by EPR, demonstrating that $\mathrm{O}_{2}^{-}$production was significantly increased compared to control group $(0.09 \pm 0.01$ vs. $0.06 \pm 0.01 \mu \mathrm{mol}$ $\mathrm{mg}^{-1} \mathrm{~min}^{-1}$, respectively, $\left.p=0.037\right)$ in the gastrocnemius.

This result was confirmed in the graph $\mathrm{C}$ representing $\mathrm{DHE}$ staining. Indeed, ROS content was increased after IR as compared to the control group $(36,860 \pm 8127$ vs. $16,720 \pm 3674 \mathrm{UA}$, respectively, $p=0.04)$.

\section{Antioxidant Defenses (Figure 3D)}

The reduced glutathione produced in gastrocnemius was critically decreased after IR as compared to the control group $(0.63 \pm 0.05$ vs. $0.45 \pm 0.04 \mu \mathrm{mol} \mathrm{L}^{-1} \mathrm{~g}^{-1}, p=0.024$, respectively).

\section{Effects of IR on the Oxidative Soleus Muscle (Figure 4) Mitochondrial Respiratory Chain Complex Activities (Figure 4A)}

Less-IR induced damage is observed in oxidative muscle. In soleus, the maximal respiration rate $\left(\mathrm{V}_{\max }\right)$, although showing a slight tendency to decrease, was not significantly altered after IR as compared to the control group $(8.94 \pm 0.60 \mathrm{vs}$. $9.56 \pm 0.58 \mu \mathrm{mol} \mathrm{O} \mathrm{min}^{-1} \mathrm{~g}^{-1}$ dry weight, respectively, $p=0.469)$.

Similarly, $\mathrm{V}_{\text {succ }}$, was not significantly modified after IR (8.99 \pm 0.43 vs. $10.05 \pm 0.52 \mu \mathrm{mol} \mathrm{O}_{2} \min ^{-1} \mathrm{~g}^{-1}$ dry weight, respectively, $p=0.135$ ).

$\mathrm{V}_{\text {TMPD/Asc }}$, showed the same profile and was not significantly altered after IR, as compared to the control group (22.70 \pm 1.32 vs. $25.88 \pm 1.92 \mu \mathrm{mol} \mathrm{O}_{2} \mathrm{~min}^{-1} \mathrm{~g}^{-1}$ dry weight, respectively, $p=$ $0.187)$.

\section{ROS Production (Figures 4B,C)}

Concerning the oxidative soleus, the graph $\mathrm{B}$ shows that $\mathrm{O}_{2}^{-}$ production was not modified as compared to the control group (0.24 $\pm 0.01 v$ s. $0.22 \pm 0.01 \mu \mathrm{mol} \mathrm{mg}^{-1} \mathrm{~min}^{-1}$, respectively, $p=$ 0.36 ).

Further, the graph C confirmed that ROS content remained unchanged after IR as compared to the control group $(109,300 \pm 17,650$ vs. $104,800 \pm 10,640$ UA, $p=0.83$, respectively).

\section{Antioxidant Defenses (Figure 4D)}

In the soleus, IR did not altered the reduced glutathione content $\left(1.14 \pm 0.13 v\right.$ s. $1.24 \pm 0.08 \mu \mathrm{mol} \mathrm{L}^{-1} \mathrm{~g}^{-1}, p=0.54$, as compared to the control group).

Taken together, these data demonstrate that unlike the glycolytic muscle gastrocnemius, the oxidative soleus is well-protected from IR-induced deleterious effects.

Thus, the alteration of $\mathrm{V}_{\max }$ was markedly greater in gastrocnemius than in soleus $(-30.28 \pm 6.17$ vs. $-6.44 \pm 6.30 \%$, respectively, $p=0.014)$. Similarly, the alteration of $\mathrm{V}_{\text {succ }}$ and of $\mathrm{V}_{\mathrm{TMPD}}$ /Asc were largely enhanced in gastrocnemius compared to soleus ( $-38.28 \pm 5.31$ vs. $-10.54 \pm 4.30 \%$, respectively, $p=$ $0.0006)$ and $(-32.71 \pm 8.48 v s .-12.29 \pm 5.08 \%, p=0.052)$, respectively (Figure $\mathbf{5 A}$ ).

Such lack of oxidative skeletal muscle alteration was associated with an absence of oxidative stress.

Accordingly, ROS production increased more in gastrocnemius than in soleus $(+39.80 \pm 16.32$ vs. $+8.36 \pm$ $6.99 \%$ in gastrocnemius and soleus, respectively, $p=0.088$, Figure 5B).

Similar data were obtained when using DHE staining $(+79.15 \pm 28.72$ vs. $+4.32 \pm 16.84 \%$ of alteration in gastrocnemius and soleus, respectively, $p=0.034$, Figure 5C).

Finally, the antioxidant defenses were reduced by IR only in the gastrocnemius as compared to the soleus $(-28.20$ \pm 6.80 vs. $-8.07 \pm 10.84 \%$, respectively, $p=0.148$, Figure 5D). 
A

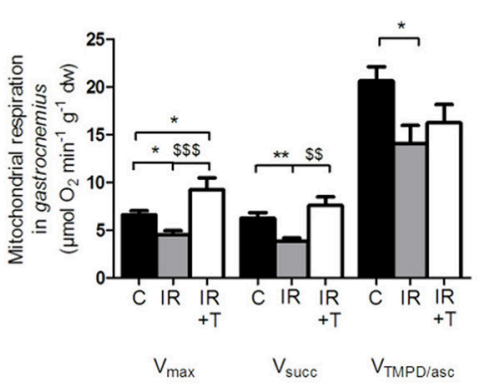

B
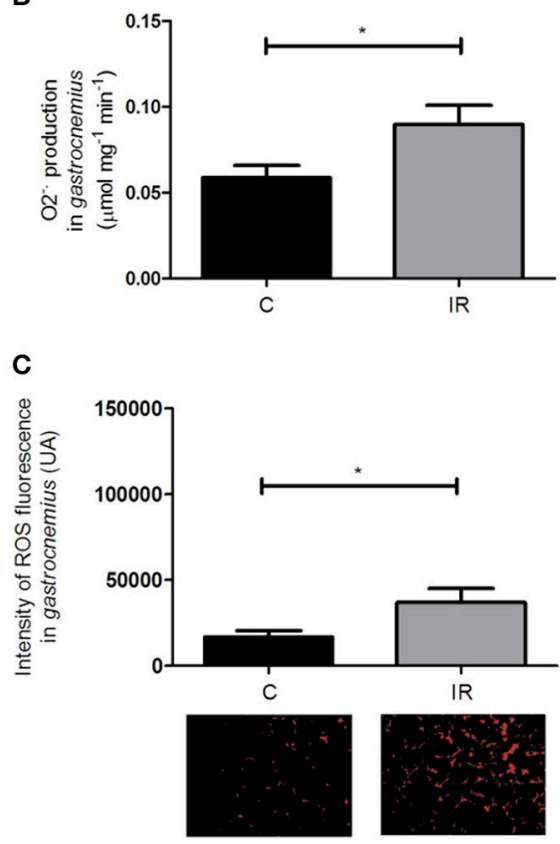

D

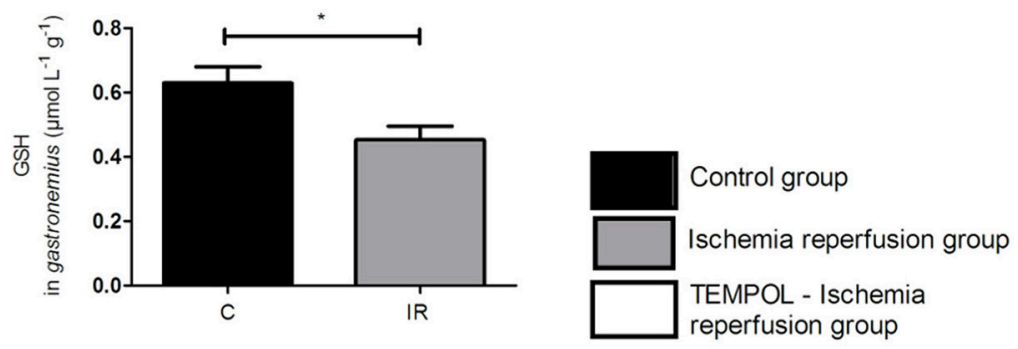

FIGURE 3 | Ischemia-reperfusion impaired glycolytic gastrocnemius muscle, and an increase of antioxidant protected this muscle against an IR injury. (A) Mitochondrial respiration in the gastrocnemius. (B,C) ROS production in the gastrocnemius: superoxide anion production measured by EPR with a specific probe $\mathrm{CMH}$ (B) and intensity of fluorescence labeled with oxidative dye DHE (C). (D) Reduced glutathione in the gastrocnemius. The black bars represent the $\mathrm{C}$ group (control group) and the gray bars represent the IR group (3 h of ischemia and $2 \mathrm{~h}$ of reperfusion). Vmax: complexes I, III, IV, V activities; Vsucc: complexes II, III, IV, V activities; $V_{T M P D / a s c}$ : complex IV activity. Results were expressed as mean \pm SEM. ${ }^{*} p<0.05,{ }^{* *} p<0.01$ compared to the control group. ${ }^{\$ \$} p<0.01,{ }^{\$ \$ \$} p<0.001$ compared to the IR group. C, Control group; IR, Ischemia-reperfusion group. IR+T: TEMPOL-IR group.

\section{Metabolic Phenotypes Implications in Muscle Susceptibility to IR}

The metabolic phenotype differs between gastrocnemius and soleus muscles and might therefore explain their specific sensitivity to IR.
Indeed, as compared to oxidative ones, glycolytic muscles are characterized by lower mitochondrial respiratory chain complex activities. This holds true for all complexes. Thus, $\mathrm{V}_{\text {max }}, \mathrm{V}_{\text {succ }}$, and $\mathrm{V}_{\mathrm{TMPD} / \mathrm{Asc}}$ are smaller in gastrocnemius as compared to soleus $(6.6 \pm 0.4$ vs. $9.6 \pm 0.6 ; 6.2$ 


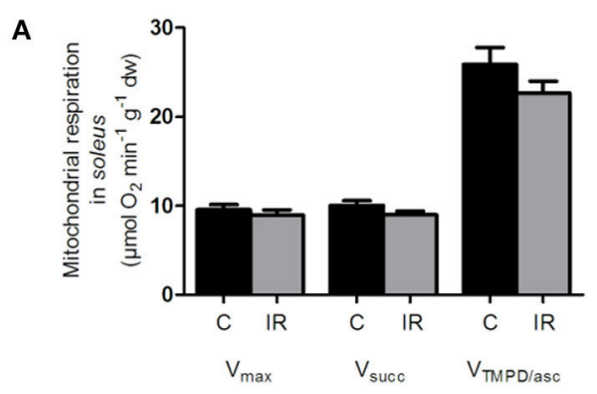

B
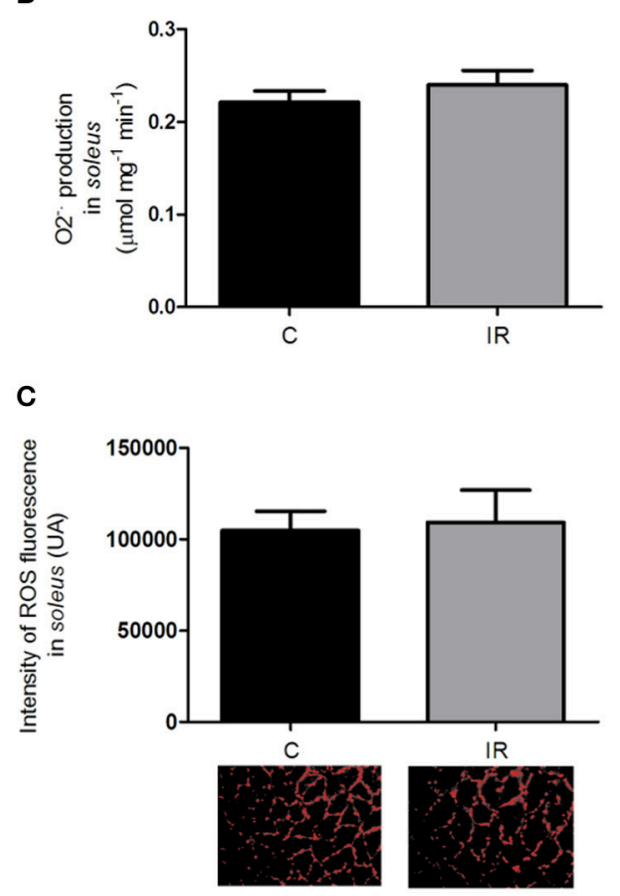

D

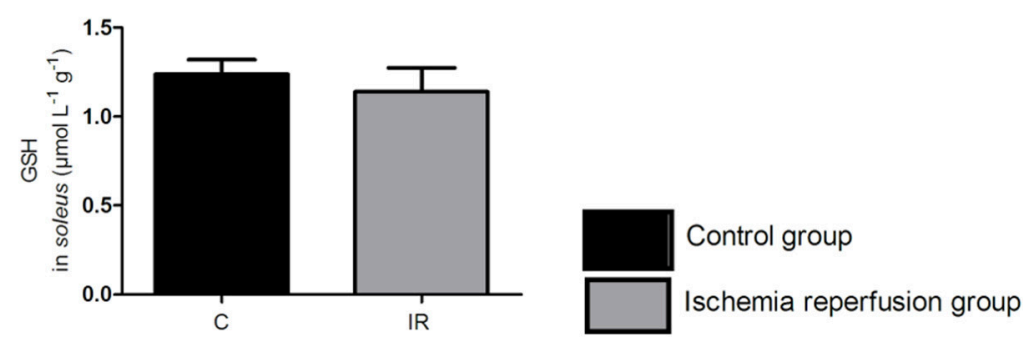

FIGURE 4 | Ischemia-reperfusion effects on the oxidative soleus muscle. (A) Mitochondrial respiration in the soleus. (B,C) ROS production in the soleus: superoxide anion production measured by EPR with a specific probe $\mathrm{CMH}$ (B) and intensity of fluorescence labeled with oxidative dye DHE (C). (D) Reduced glutathione in the soleus. The black bars represent the $\mathrm{C}$ group (control group) and the gray bars represent the IR group ( $3 \mathrm{~h}$ of ischemia and $2 \mathrm{~h}$ of reperfusion). $\mathrm{V}_{\text {max }}$ : complexes I, III, IV, V activities; Vsucc: complexes II, III, IV, V activities; VTMPD/asc: complex IV activity. Results were expressed as mean \pm SEM. C, Control group, IR, Ischemia-reperfusion group.

\pm 0.6 vs. $10.0 \pm 0.5$; and $20.6 \pm 1.5$ vs. $25.9 \pm 1.9$ $\mu \mathrm{mol} \mathrm{O}_{2} \min ^{-1} \mathrm{~g}^{-1}$ dry weight, respectively, $p<0.001$ ) (Figures 3A, 4A).

Further, $\mathrm{O}_{2}^{-}$production determined by EPR was significantly lower in gastrocnemius than in soleus $(0.059$ $\pm 0.007,0.221 \pm 0.012 \mu \mathrm{mol} \mathrm{mg} \mathrm{m}^{-1} \mathrm{~min}^{-1}$, respectively, $p<0.001$, Figures 3B, 4B). This is confirmed by DHE staining demonstrating that ROS level was a six-fold lower in gastrocnemius as compared to soleus $(16,720 \pm 3674$ vs. 104,800 $\pm 10,640 \mathrm{UA}, p<0.001$, respectively, Figures 3C, 4C). 
A

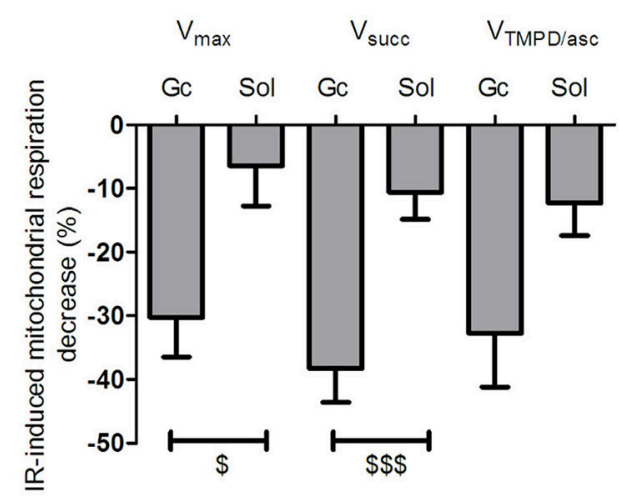

B

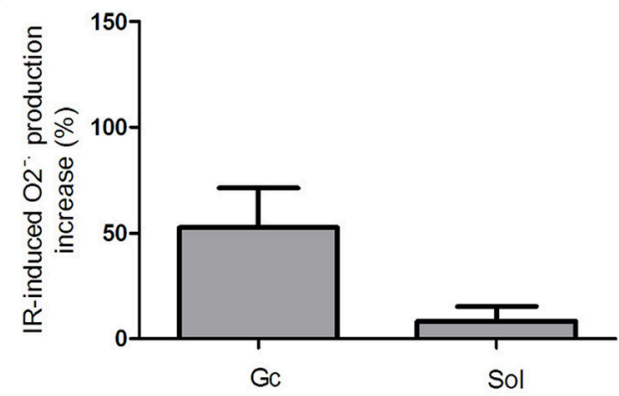

C

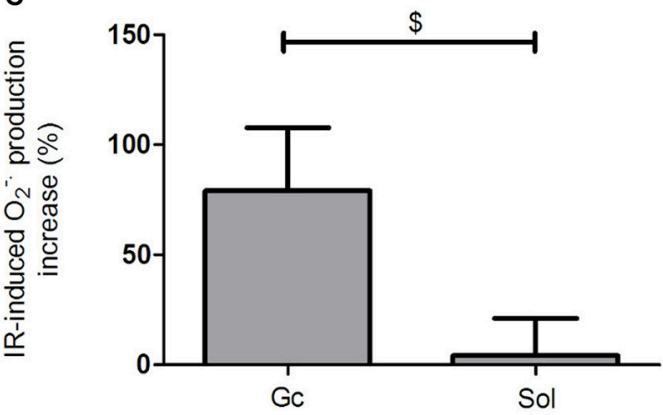

D

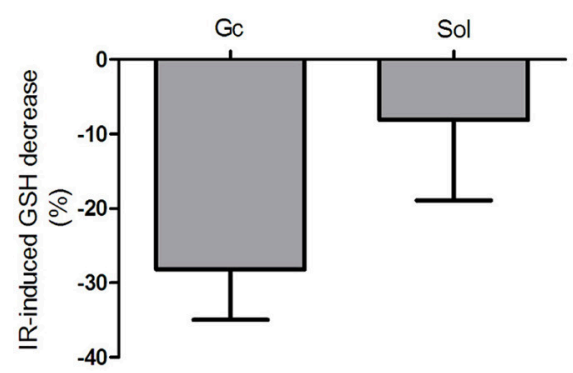

FIGURE 5 | Ischemia reperfusion altered specifically glycolytic compared to oxidative skeletal muscles. (A) IR-induced mitochondrial respiration in the gastrocnemius (Gc) compared to the soleus (Sol) expressed in \%. (B,C) ROS production in the gastrocnemius (Gc) compared to the soleus (Sol) expressed in \%. Superoxide anion production measured by EPR with a specific probe $\mathrm{CMH}$ (B) and intensity of fluorescence labeled with oxidative dye DHE (C). (D) The use of IR-induced reduced glutathione was compared in gastrocnemius (Gc) and soleus (Sol), and expressed in \%. Results were expressed as mean \pm SEM. ${ }^{\$} p<0.05,{ }^{\$ \$ \$} p<0.001$ compared to gastrocnemius.
Finally, gastrocnemius muscle antioxidant defenses, as inferred from GSH are two-fold lower in gastrocnemius compared to soleus $\left(0.63 \pm 0.05\right.$ vs. $1.24 \pm 0.08 \mu \mathrm{mol} \mathrm{L}^{-1} \mathrm{~g}^{-1}$, $p<0.0001$, respectively, Figures 3D, 4D).

In view of these data we tested the hypothesis that the reduced antioxidant defenses of gastrocnemius muscle might be overcome by administration of tempol, an efficient antioxidant, and therefore confer protection against IR to this glycolytic muscle.

With tempol, mitochondrial oxidative capacities were no longer impaired by IR in gastrocnemius (Figure 3A).

Thus, in the Tempol-IR group, $\mathrm{V}_{\max }$ was not impaired by IR $\left(9.25 \pm 1.25, v s .3 .82 \pm 0.36 \mu\right.$ mol $\mathrm{O}_{2} \min ^{-1} \mathrm{~g}^{-1}$ dry weight, $p<$ 0.001 and $6.23 \pm 0.61 \mu \mathrm{mol} \mathrm{O}_{2} \min ^{-1} \mathrm{~g}^{-1}$ dry weight, $p<0.05$, for IR and control group, respectively).

Similarly, $\mathrm{V}_{\text {succ }}$ was not impaired by IR $(7.58 \pm 0.90$ vs. 3.82 $\pm 0.36 \mu \mathrm{mol} \mathrm{O}_{2} \mathrm{~min}^{-1} \mathrm{~g}^{-1}$ dry weight, $p<0.01$, and $6.23 \pm 0.61$ $\mu \mathrm{mol} \mathrm{O}_{2} \mathrm{~min}^{-1} \mathrm{~g}^{-1}$ dry weight, $p=\mathrm{NS}$ for IR and control group, respectively). $\mathrm{V}_{\mathrm{TMPD} / \text { asc }}(16.24 \pm 1.92)$ was not significantly different from IR and control values $(14.05 \pm 1.92$ and $20.61 \pm$ $1.49 \mu \mathrm{mol} \mathrm{O}_{2} \mathrm{~min}^{-1} \mathrm{~g}^{-1}$ dry weight, for IR and control group, respectively).

\section{DISCUSSION}

The main result of this study is that skeletal muscle metabolic phenotype importantly modulates deleterious effects of lower limb IR. Thus, as compared to the glycolytic gastrocnemius, the oxidative soleus appeared protected and demonstrated no significant mitochondrial dysfunction. In soleus, higher antioxidant capacity likely participates in such protection since oxidative stress is a key factor inducing muscle impairment during IR. Accordingly, when treated with an antioxidant, gastrocnemius muscle acted as soleus, showing no more significant alteration although submitted to the same IR protocol.

Vascular ischemia is very common and corresponds to a public health issue both because of its increasing occurrence and its very bad prognosis (Yassin et al., 2002). Thus, major amputation is still needed in $25-40 \%$ of cases, and the risk of death is as high as $25 \%$ after the first clinical sign of peripheral arteriopathy (Norgren et al., 2007; Taylor et al., 2009; Ryan et al., 2015). Interestingly, muscle alteration significantly impacts both local and general prognosis. Thus, rhabdomyolysis is well-known to participate in remote organ alteration including kidneys, the heart and lungs (Fowkes et al., 2006; Ali et al., 2007; McMahon et al., 2013).

Although some therapeutic strategies have been shown effective, results are not always consistent, raising the issue of a specific organ dependency to IR (Mansour et al., 2014). Concerning skeletal muscles, metabolic phenotype is a good candidate since degrees of fiber injury vary among contractile muscle phenotypes (Chan et al., 2004). Woitaske et al. observed an increased mitochondrial swelling and disrupted sarcoplasmic reticulum in the glycolytic EDL as compared to soleus muscle (Woitaske and McCarter, 1998). Accordingly, IR- induced 
contractile dysfunction was recently shown to be reduced in soleus as compared to EDL (Demirel et al., 2013).

Based on different muscle phenotypes, several mechanisms might explain such potential increased sensitivity of glycolytic muscle to IR injury. Particularly, mitochondrial content and redox balance deserve discussion.

Mitochondrial density is about two-fold greater in oxidative than in glycolytic muscles (Jackman and Willis, 1996; Picard et al., 2008), likely participating in the enhanced mitochondrial respiration observed in soleus as compared to gastrocnemius muscles (Anderson and Neufer, 2006; Meyer et al., 2014). Recently, induction of mitochondrial biogenesis has been shown to protect against apoptosis in L6 myoblats (Dam et al., 2013), and we observed that decrease in PGC1 beta, which likely participate in mitochondrial biogenesis, resulted in a reduced adaptation of muscles to cope with high energy demands (Gali Ramamoorthy et al., 2015). Such relationship between the number of mitochondria and muscle responses to stress likely apply in the setting of IR. Accordingly, Flück et al. demonstrated that IR-induced skeletal muscle rhabdomyolysis is a fiber type-specific phenomenon, modulated by mitochondria reserves (Fluck et al., 2015).

However, besides mitochondrial respiration per-se, redox status appears to be a key factor in muscle functioning (for review see Zuo and Pannell, 2015). Even during physiological contraction (eccentric and/or concentric), muscle mitochondria generate large amounts of ROS, that might allow either muscular adaptation or muscular damage. Thus, depending likely on ROS levels resulting from the pro-/antioxidant balance, consequences might be good (mitochondrial biogenesis, antioxidant pathway stimulation, enhanced glucose uptake), bad (mitochondrial dysfunctions, reduced ATP synthesis) or even ugly (mPTP opening and apoptosis) (Lejay et al., 2014; Zuo et al., 2015).

In this view, exercise is interesting to consider. Indeed, exercise-related beneficial effects are largely reduced when exercise-induced ROS signaling is decreased by enzymatic inhibitors or antioxidant supplementation (Meier et al., 2013; Strobel et al., 2014; Merry and Ristow, 2016). Further, the effects of exercise likely depend on muscle metabolic phenotype. For instance, exercise has a stronger influence on red muscle phenotype, as compared to white muscle in salmonids (Morash et al., 2014). Similarly, uphill-, as compared to down-hill training enhanced mitochondrial function specifically in both soleus and vastus intermedius skeletal muscle (Schlagowski et al., 2016).

Increased antioxidant defenses also characterize oxidative muscles. This likely explains why statins optimize cardiac mitochondria, but impairs skeletal muscle function by inducing different levels of ROS (Bouitbir et al., 2011, 2015). Concerning skeletal muscles, in agreement with previous reports (Anderson and Neufer, 2006; Picard et al., 2012), we observed greater

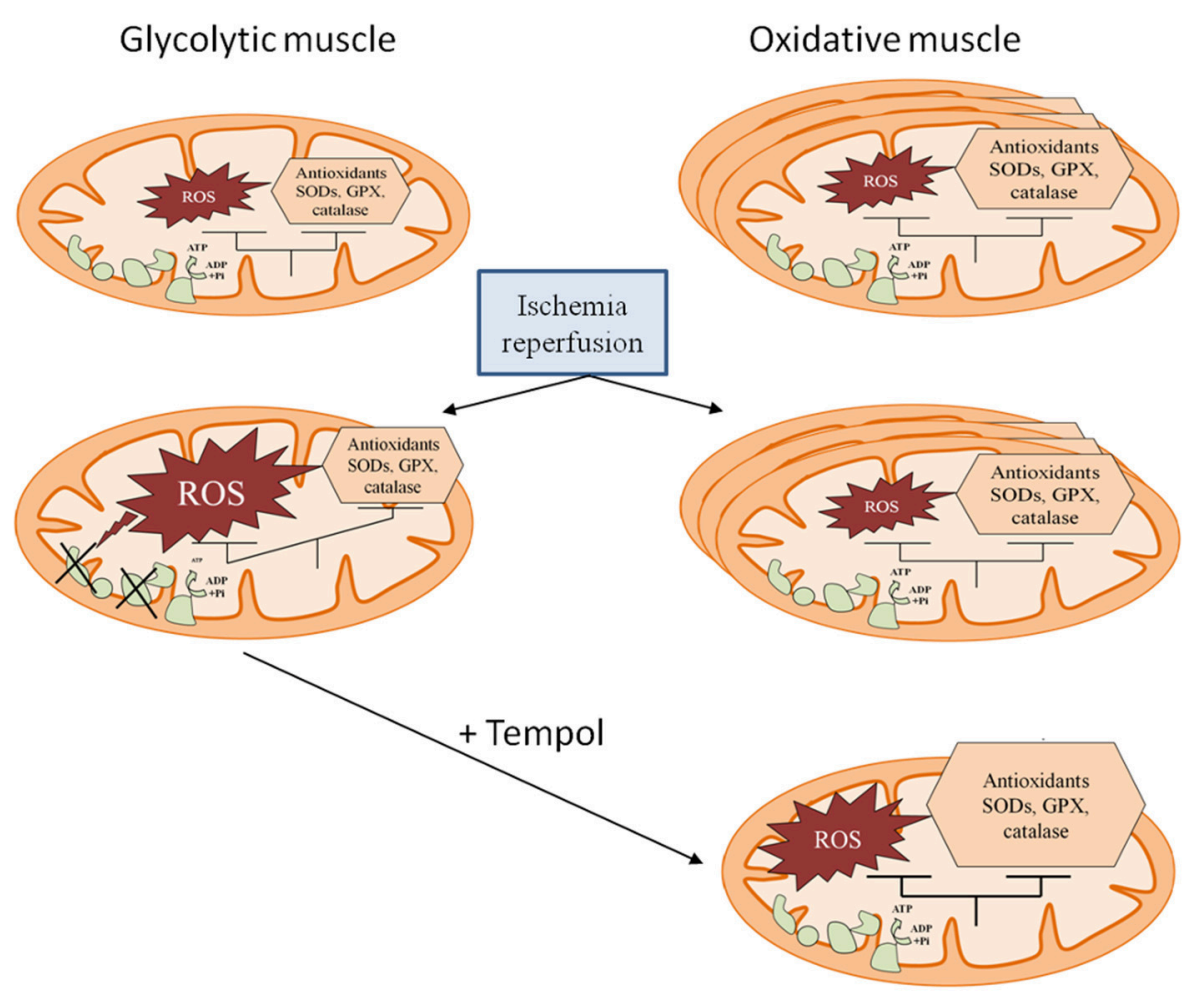

FIGURE 6 | Schema illustrating skeletal muscle susceptibility to ischemia-reperfusion, depending on metabolic phenotype. Oxidative skeletal muscles are characterized by increased mitochondrial content and enhanced antioxidant system allowing better protection against ischemia-reperfusion supported by maintained mitochondrial respiration and low reactive oxygen species (ROS) production. Antioxidant adjunction protects glycolytic muscle, further demonstrating the key role of oxidative stress handling and therefore muscle metabolic phenotype. SODs, Superoxide dismutases; GPX, glutathione peroxidase. 
glutathione peroxidase activity in the soleus than in the superficial gastrocnemius This support that the oxidative skeletal muscle may possess a greater capacity to scavenge ROS (Picard et al., 2012). Since enhanced ROS production occur early and participate significantly in IR deleterious effects (Tran et al., 2011; Guillot et al., 2014; Lejay et al., 2014), the fact that oxidative muscles naturally equip with higher antioxidant contents than glycolytic muscles likely protect them in the setting of IR. Further, an adaptation related to oxidative resistance might have been triggered during IR. Such a possibility, likely working in the setting of preconditioning, can perhaps not be totally developed in the relatively short time frame of our study but, one might suggest that the antioxidant pool located in the blood might also have been mobilized.

To further test that enhanced antioxidant defenses allow oxidative skeletal muscles being protected against IR, we determined whether the glycolytic muscle gastrocnemius might be protected against IR when its antioxidant defense is improved. Accordingly, tempol therapy significantly attenuated the deleterious effects of IR. Such data are in agreement with a previous work (Tran et al., 2011) and further suggest a key role of the antioxidant defenses and likely explain -at least partly- the specific muscle susceptibility to IR (Figure 6).

As perspectives, further studies should be able to demonstrate that ROS staining be mainly colocalized within type II fibers during IR. It might be useful to determine the molecular mechanisms involved in muscle protection, signaling ranging from mitochondrial biogenesis to atrophy-hypertrophy pathways. Besides ROS-activated signaling pathways such as MAPK, PI3K/Akt signaling, PKC, and inflammation signaling (Görlach et al., 2015), a special insight should be directed toward the SAFE and RISK pathways which modified interplay

\section{REFERENCES}

Akerboom, T. P., and Sies, H. (1981). Assay of glutathione, glutathione disulfide, and glutathione mixed disulfides in biological samples. Methods Enzymol. 77, 373-382. doi: 10.1016/S0076-6879(81)77050-2

Ali, M., Cho, W. J., Hudson, B., Kassiri, Z., Granzier, H., and Schulz, R. (2010). Titin is a target of matrix metalloproteinase-2: implications in myocardial ischemia/reperfusion injury. Circulation 122, 2039-2047. doi: 10.1161/CIRCULATIONAHA.109.930222

Ali, Z. A., Callaghan, C. J., Lim, E., Ali, A. A., Nouraei, S. A., Akthar, A. M., et al. (2007). Remote ischemic preconditioning reduces myocardial and renal injury after elective abdominal aortic aneurysm repair: a randomized controlled trial. Circulation 116, I98-105. doi: 10.1161/circulationaha.106. 679167

Anderson, E. J., and Neufer, P. D. (2006). Type II skeletal myofibers possess unique properties that potentiate mitochondrial $\mathrm{H}(2) \mathrm{O}(2)$ generation. Am. J. Physiol. Cell Physiol. 290, C844-C851. doi: 10.1152/ajpcell.00402.2005

Antunes, F., Han, D., and Cadenas, E. (2002). Relative contributions of heart mitochondria glutathione peroxidase and catalase to $\mathrm{H}(2) \mathrm{O}(2)$ detoxification in in vivo conditions. Free Radic. Biol. Med. 33, 1260-1267. doi: 10.1016/S0891-5849(02)01016-X

Avci, G., Kadioglu, H., Sehirli, A. O., Bozkurt, S., Guclu, O., Arslan, E., et al. (2012). Curcumin protects against ischemia/reperfusion injury in rat skeletal muscle. J. Surg. Res. 172, e39-e46. doi: 10.1016/j.jss.2011.08.021

Balogh, A., Santer, D., Pásztor, E., Tóth, A., Czuriga, D., Podesser, B., et al. (2014). Myofilament protein carbonylation contributes to the contractile dysfunction might account for increased susceptibility of diabetic muscles to ischemia (Lejay et al., 2016).

\section{CONCLUSION}

This study supports that glycolytic muscles are more prone to IR than oxidative ones. Such muscle susceptibility appears linked to muscle metabolic phenotypes, involving mainly muscle mitochondrial oxidative capacity and antioxidant defenses. This might explain difficulties to convert experimental data well-focused on specific muscle fibers toward clinical setting, since human muscles show more phenotypic heterogeneity. More widely, these data suggest that therapeutic approaches should be adapted to target organ redox capacities (potentially modified by comorbidities), and that mechanisms increasing either mitochondrial function and/or antioxidant defense should allow better protection.

\section{AUTHOR CONTRIBUTIONS}

Conception or design of the work: AC, AG, MG, ST, AL, JB, BG. Acquisition, analysis: AC, AG, MG, ST, AL, JB, JZ. Interpretation of data for the work: AC, AG, AM, MK, VW, JZ, BG. Drafting or revising the work: AC, AG, MG, VW, JB, JZ, BG. Final approval: AC, AG, MG, ST, AL, AM, MK, VW, JB, JZ, BG. Agreement to be accountable of all aspects of the work: AC, AG, MG, ST, AL, AM, $\mathrm{MK}, \mathrm{VW}, \mathrm{JB}, \mathrm{JZ}, \mathrm{BG}$.

\section{ACKNOWLEDGMENTS}

The authors thank F. Goupilleau, I. Bentz, and A. M. Kasprowicz for excellent technical and secretarial assistances. in the infarcted LV region of mouse hearts. Cardiovasc. Res. 101, 108-119. doi: $10.1093 / \mathrm{cvr} / \mathrm{cvt} 236$

Beckendorf, L., and Linke, W. (2015). Emerging importance of oxidative stress in regulating striated muscle elasticity. J. Muscle Res. Cell Motil. 36, 25-36. doi: 10.1007/s10974-014-9392-y

Beekley, A. C., Sebesta, J. A., Blackbourne, L. H., Herbert, G. S., Kauvar, D. S., Baer, D. G., et al. (2008). Prehospital tourniquet use in Operation Iraqi Freedom: effect on hemorrhage control and outcomes. J. Trauma 64, S28-S37. discussion: S37. doi: 10.1097/ta.0b013e318160937e

Belkin, M., Brown, R. D., Wright, J. G., LaMorte, W. W., and Hobson, R. W. II. (1988). A new quantitative spectrophotometric assay of ischemia-reperfusion injury in skeletal muscle. Am. J. Surg. 156, 83-86. doi: 10.1016/S0002-9610(88)80360-X

Blaauw, B., Schiaffino, S., and Reggiani, C. (2013). Mechanisms modulating skeletal muscle phenotype. Compr. Physiol. 3, 1645-1687. doi: 10.1002/cphy.c130009

Blaisdell, F. W. (2002). The pathophysiology of skeletal muscle ischemia and the reperfusion syndrome: a review. Cardiovasc. Surg. 10, 620-630. doi: 10.1016/S0967-2109(02)00070-4

Bloemberg, D., and Quadrilatero, J. (2012). Rapid determination of myosin heavy chain expression in rat, mouse, and human skeletal muscle using multicolor immunofluorescence analysis. PLoS ONE 7:e35273. doi: 10.1371/journal.pone.0035273

Bouitbir, J., Charles, A. L., Echaniz-Laguna, A., Kindo, M., Daussin, F., Auwerx, J., et al. (2011). Opposite effects of statins on mitochondria of cardiac and skeletal muscles: a 'mitohormesis' mechanism involving reactive oxygen species and PGC-1. Eur. Heart J. 33, 1397-1407. doi: 10.1093/eurheartj/ehr224 
Bouitbir, J., Singh, F., Charles, A. L., Schlagowski, A. I., Bonifacio, A., Echaniz-Laguna, A., et al. (2015). Statins trigger mitochondrial ROS-induced apoptosis in glycolytic skeletal muscle. Antioxid. Redox Signal. 24, 84-98. doi: 10.1089/ars.2014.6190

Carvalho, A. J., McKee, N. H., and Green, H. J. (1997). Metabolic and contractile responses of fast and slow twitch rat skeletal muscles to ischemia and reperfusion. Plast. Reconstr. Surg. 99, 163-171. doi: 10.1097/00006534-199701000-00025

Chan, R. K., Austen, W. G. Jr., Ibrahim, S., Ding, G. Y., Verna, N., Hechtman, H. B., et al. (2004). Reperfusion injury to skeletal muscle affects primarily type II muscle fibers. J. Surg. Res. 122, 54-60. doi: 10.1016/j.jss.2004.05.003

Charles, A. L., Guilbert, A. S., Bouitbir, J., Goette-Di Marco, P., Enache, I., Zoll, J., et al. (2011). Effect of postconditioning on mitochondrial dysfunction in experimental aortic cross-clamping. Br. J. Surg. 98, 511-516. doi: $10.1002 /$ bjs. 7384

Dam, A. D., Mitchell, A. S., and Quadrilatero, J. (2013). Induction of mitochondrial biogenesis protects against caspase-dependent and caspaseindependent apoptosis in L6 myoblasts. Biochim. Biophys. Acta 1833, 3426-3435. doi: 10.1016/j.bbamcr.2013.04.014

Demirel, M., Kaya, B., Cerkez, C., Ertunc, M., and Sara, Y. (2013). Lcarnitine pretreatment protects slow-twitch skeletal muscles in a rat model of ischemia-reperfusion injury. Vasc. Endovascular Surg. 47, 540-545. doi: $10.1177 / 1538574413496481$

Dikalov, S., Griendling, K. K., and Harrison, D. G. (2007). Measurement of reactive oxygen species in cardiovascular studies. Hypertension 49, 717-727. doi: 10.1161/01.HYP.0000258594.87211.6b

Fluck, M., von Allmen, R. S., Ferrie, C., Tevaearai, H., and Dick, F. (2015). Protective effect of focal adhesion kinase against skeletal muscle reperfusion injury after acute limb ischemia. Eur. J. Vasc. Endovasc. Surg. 49, 306-313. doi: 10.1016/j.ejvs.2014.11.011

Fowkes, F. G., Anandan, C. L., Lee, A. J., Smith, F. B., Tzoulaki, I., Rumley, A., et al. (2006). Reduced lung function in patients with abdominal aortic aneurysm is associated with activation of inflammation and hemostasis, not smoking or cardiovascular disease. J. Vasc. Surg. 43, 474-480. doi: 10.1016/j.jvs.2005.11.018

Gali Ramamoorthy, T., Laverny, G., Schlagowski, A., Zoll, J., Messaddeq, N., Bornert, J. M., et al. (2015). The transcriptional coregulator PGC-1b controls mitochondrial function and anti-oxidant defence in skeletal muscles. Nat. Commun. 17:10210. doi: 10.1038/ncomms10210

Görlach, A., Dimova, E., Petry, A., Martínez-Ruiz, A., Hernansanz-Agustín, P., Rolo, A. P., et al. (2015). Reactive oxygen species, nutrition, hypoxia and diseases: Problems solved? Redox Biol. 6, 372-385. doi: 10.1016/j.redox.2015.08.016

Guillot, M., Charles, A. L., Chamaraux-Tran, T. N., Bouitbir, J., Meyer, A., Zoll, J., et al. (2014). Oxidative stress precedes skeletal muscle mitochondrial dysfunction during experimental aortic cross-clamping but is not associated with early lung, heart, brain, liver, or kidney mitochondrial impairment. J. Vasc. Surg. 60, 1043-1051. doi: 10.1016/j.jvs.2013.07.100

Halter, B., Gonzalez de Aguilar, J. L., Rene, F., Petri, S., Fricker, B., Echaniz-Laguna, A., et al. (2010). Oxidative stress in skeletal muscle stimulates early expression of Rad in a mouse model of amyotrophic lateral sclerosis. Free Radic. Biol. Med. 48, 915-923. doi: 10.1016/j.freeradbiomed.2010.01.014

Harris, K., Walker, P. M., Mickle, D. A., Harding, R., Gatley, R., Wilson, G. J., et al. (1986). Metabolic response of skeletal muscle to ischemia. Am. J. Physiol. 250, $\mathrm{H} 213-\mathrm{H} 220$.

He, F., and Zuo, L. (2015). Redox roles of reactive oxygen species in cardiovascular diseases. Int. J. Mol. Sci. 16, 27770-27780. doi: 10.3390/ijms161126059

Itoh, M., Shimokawa, N., Tajika, Y., Murakami, T., Aotsuka, N., Lesmana, R., et al. (2013). Alterations of biochemical marker levels and myonuclear numbers in rat skeletal muscle after ischemia-reperfusion. Mol. Cell. Biochem. 373, 11-18. doi: 10.1007/s11010-012-1470-0

Jackman, M. R., and Willis, W. T. (1996). Characteristics of mitochondria isolated from type I and type IIb skeletal muscle. Am. J. Physiol. 270, C673-C678.

Kindo, M., Gerelli, S., Bouitbir, J., Charles, A. L., Zoll, J., Hoang Minh, T., et al. (2012). Pressure overload-induced mild cardiac hypertrophy reduces left ventricular transmural differences in mitochondrial respiratory chain activity and increases oxidative stress. Front. Physiol. 3:332. doi: 10.3389/fphys.2012.00332
Kindo, M., Gerelli, S., Bouitbir, J., Hoang Minh, T., Charles, A. L., Mazzucotelli, J. P., et al. (2016). Left ventricular transmural gradient in mitochondrial respiration is associated with increased sub-endocardium nitric oxide and reactive oxygen species productions. Front. Physiol. 7:331. doi: 10.3389/fphys.2016.00331

Kowaltowski, A. J., de Souza-Pinto, N. C., Castilho, R. F., and Vercesi, A. E. (2009). Mitochondria and reactive oxygen species. Free Radic. Biol. Med. 47, 333-343. doi: 10.1016/j.freeradbiomed.2009.05.004

Kuznetsov, A. V., Kehrer, I., Kozlov, A. V., Haller, M., Redl, H., Hermann, M., et al. (2011). Mitochondrial ROS production under cellular stress: comparison of different detection methods. Anal. Bioanal. Chem. 400, 2383-2390. doi: 10.1007/s00216-011-4764-2

Kuznetsov, A. V., Veksler, V., Gellerich, F. N., Saks, V., Margreiter, R., and Kunz, W. S. (2008). Analysis of mitochondrial function in situ in permeabilized muscle fibers, tissues and cells. Nat. Protoc. 3, 965-976. doi: 10.1038/nprot.2008.61

Lejay, A., Choquet, P., Thaveau, F., Singh, F., Schlagowski, A., Charles, A. L., et al. (2015). A new murine model of sustainable and durable chronic critical limb ischemia fairly mimicking human pathology. Eur. J. Vasc. Endovasc. Surg. 49, 205-212. doi: 10.1016/j.ejvs.2014.12.010

Lejay, A., Fang, F., John, R., Van, J. A., Barr, M., Thaveau, F., et al. (2016). Ischemia reperfusion injury, ischemic conditioning and diabetes mellitus. J. Mol. Cell Cardiol. 91, 11-22. doi: 10.1016/j.yjmcc.2015.12.020

Lejay, A., Meyer, A., Schlagowski, A. I., Charles, A. L., Singh, F., Bouitbir, J., et al. (2014). Mitochondria: mitochondrial participation in ischemiareperfusion injury in skeletal muscle. Int. J. Biochem. Cell Biol. 50, 101-105. doi: 10.1016/j.biocel.2014.02.013

Ma, L., Chuang, C., Weng, W., Zhao, L., Zheng, Y., Zhang, J., et al. (2016). Paeonol protects rat heart by improving regional blood perfusion during no-reflow. Front. Physiol. 7:298. doi: 10.3389/fphys.2016.00298

Magder, S. (2006). Reactive oxygen species: toxic molecules or spark of life? Crit. Care 10, 208. doi: 10.1186/cc3992

Makris, K. I., Nella, A. A., Zhu, Z., Swanson, S. A., Casale, G. P., Gutti, T. L., et al. (2007). Mitochondriopathy of peripheral arterial disease. Vascular 15, 336-343. doi: 10.2310/6670.2007.00054

Mansour, Z., Bouitbir, J., Charles, A. L., Talha, S., Kindo, M., Pottecher, J., et al. (2011). Remote and local ischemic preconditioning equivalently protects rat skeletal muscle mitochondrial function during experimental aortic cross-clamping. J. Vasc. Surg. 55, 497-505.e1. doi: 10.1016/j.jvs.2011. 07.084

Mansour, Z., Charles, A. L., Kindo, M., Pottecher, J., Chamaraux-Tran, T. N., Lejay, A., et al. (2014). Remote effects of lower limb ischemia-reperfusion: impaired lung, unchanged liver, and stimulated kidney oxidative capacities. Biomed Res. Int. 2014:392390. doi: 10.1155/2014/392390

Martou, G., O’Blenes, C. A., Huang, N., McAllister, S. E., Neligan, P. C., Ashrafpour, H., et al. (2006). Development of an in vitro model for study of the efficacy of ischemic preconditioning in human skeletal muscle against ischemia-reperfusion injury. J. Appl. Physiol. 101, 1335-1342. doi: 10.1152/japplphysiol.00278.2006

Masuda, K., Tanabe, K., Kuno, S., Hirayama, A., and Nagase, S. (2003). Antioxidant capacity in rat skeletal muscle tissues determined by electron spin resonance. Comp. Biochem. Physiol. B Biochem. Mol. Biol. 134, 215-220. doi: 10.1016/S1096-4959(02)00254-3

McMahon, G. M., Zeng, X., and Waikar, S. S. (2013). A risk prediction score for kidney failure or mortality in rhabdomyolysis. JAMA Intern. Med. 173, 1821-1828. doi: 10.1001/jamainternmed.2013.9774

Meier, P., Renga, M., Hoppeler, H., and Baum, O. (2013). The impact of antioxidant supplements and endurance exercise on genes of the carbohydrate and lipid metabolism in skeletal muscle of mice. Cell Biochem. Funct. 31, 51-59. doi: $10.1002 / \mathrm{cbf} .2859$

Merry, T. L., and Ristow, M. (2016). Do antioxidant supplements interfere with skeletal muscle adaptation to exercise training? J. Physiol. 594, 5135-5147. doi: 10.1113/JP270654

Meyer, A., Charles, A. L., Zoll, J., Guillot, M., Lejay, A., Singh, F., et al. (2014). Cryopreservation with dimethyl sulfoxide prevents accurate analysis of skinned skeletal muscle fibers mitochondrial respiration. Biochimie 100, 227-233. doi: 10.1016/j.biochi.2014.01.014 
Morash, A. J., Vanderveken, M., and McClelland, G. B. (2014). Muscle metabolic remodeling in response to endurance exercise in salmonids. Front. Physiol. 5:452. doi: 10.3389/fphys.2014.00452

Mulsch, A., Oelze, M., Kloss, S., Mollnau, H., Topfer, A., Smolenski, A., et al. (2001). Effects of in vivo nitroglycerin treatment on activity and expression of the guanylyl cyclase and cGMP-dependent protein kinase and their downstream target vasodilator-stimulated phosphoprotein in aorta. Circulation 103, 2188-2194. doi: 10.1161/01.CIR.103.17.2188

Noll, E., Bouitbir, J., Collange, O., Zoll, J., Charles, A. L., Thaveau, F., et al. (2012). Local but not systemic capillary lactate is a reperfusion biomarker in experimental acute limb ischaemia. Eur. J. Vasc. Endovasc. Surg. 43, 339-340. doi: 10.1016/j.ejvs.2011.12.015

Norgren, L., Hiatt, W. R., Dormandy, J. A., Nehler, M. R., Harris, K. A., Fowkes, F. G., et al. (2007). Inter-society consensus for the management of peripheral arterial disease (TASC II). Eur. J. Vasc. Endovasc. Surg. 33(Suppl. 1), S1-S75. doi: 10.1016/j.ejvs.2006.09.024

Paradis, S., Charles, A. L., Meyer, A., Lejay, A., Scholey, J. W., Chakfé, N., et al. (2016). Chronology of mitochondrial and cellular events during skeletal muscle ischemia-reperfusion. Am. J. Physiol. Cell Physiol. 310, C968-C982. doi: 10.1152/ajpcell.00356.2015

Picard, M., Csukly, K., Robillard, M. E., Godin, R., Ascah, A., BourcierLucas, C., et al. (2008). Resistance to Ca2+-induced opening of the permeability transition pore differs in mitochondria from glycolytic and oxidative muscles. Am. J. Physiol. Regul. Integr. Comp. Physiol. 295, R659-R668. doi: 10.1152/ajpregu.90357.2008

Picard, M., Hepple, R. T., and Burelle, Y. (2012). Mitochondrial functional specialization in glycolytic and oxidative muscle fibers: tailoring the organelle for optimal function. Am. J. Physiol. Cell Physiol. 302, C629-C641. doi: 10.1152/ajpcell.00368.2011

Ploquin, C., Chabi, B., Fouret, G., Vernus, B., Feillet-Coudray, C., Coudray, C., et al. (2012). Lack of myostatin alters intermyofibrillar mitochondria activity, unbalances redox status, and impairs tolerance to chronic repetitive contractions in muscle. Am. J. Physiol. Endocrinol. Metab. 302, E1000-E1008. doi: 10.1152/ajpendo.00652.2011

Pottecher, J., Guillot, M., Belaidi, E., Charles, A. L., Lejay, A., Gharib, A., et al. (2013). Cyclosporine A normalizes mitochondrial coupling, reactive oxygen species production, and inflammation and partially restores skeletal muscle maximal oxidative capacity in experimental aortic cross-clamping. J. Vasc. Surg. 57, 1100-1108.e2. doi: 10.1016/j.jvs.2012.09.020

Pourova, J., Kottova, M., Voprsalova, M., and Pour, M. (2010). Reactive oxygen and nitrogen species in normal physiological processes. Acta Physiol. 198, 15-35. doi: 10.1111/j.1748-1716.2009.02039.x

Powers, S. K., and Hamilton, K. (1999). Antioxidants and exercise. Clin. Sports Med. 18, 525-536. doi: 10.1016/S0278-5919(05)70166-6

Rasmussen, U. F., and Rasmussen, H. N. (2000). Human skeletal muscle mitochondrial capacity. Acta Physiol. Scand. 168, 473-480. doi: 10.1046/j.1365-201x.2000.00699.x

Ristow, M. (2014). Unraveling the Truth About Antioxidants: mitohormesis explains ROS-induced health benefits. Nat. Med. 20, 709-711. doi: $10.1038 / \mathrm{nm} .3624$

Ryan, T. E., Schmidt, C. A., Green, T. D., Brown, D. A., Neufer, P. D., and McClung, J. M. (2015). Mitochondrial regulation of the muscle microenvironment in critical limb ischemia. Front. Physiol. 6:336. doi: 10.3389/fphys.2015. 00336

Saks, V. A., Veksler, V. I., Kuznetsov, A. V., Kay, L., Sikk, P., Tiivel, T., et al. (1998). Permeabilized cell and skinned fiber techniques in studies of mitochondrial function in vivo. Mol. Cell. Biochem. 184, 81-100. doi: 10.1023/A:1006834912257

Schiaffino, S. (2010). Fibre types in skeletal muscle: a personal account. Acta Physiol. 199, 451-463. doi: 10.1111/j.1748-1716.2010.02130.x

Schiaffino, S., and Reggiani, C. (2011). Fiber types in mammalian skeletal muscles. Physiol. Rev. 91, 1447-1531. doi: 10.1152/physrev.00031.2010

Schlagowski, A. I., Isner-Horobeti, M. E., Dufour, S. P., Rasseneur, L., Enache, I., Lonsdorfer-Wolf, E., et al. (2016). Mitochondrial function following downhill and/or uphill exercise training in rats. Muscle Nerve 54, 925-935. doi: $10.1002 /$ mus. 25144

Strobel, N., Matsumoto, A., Peake, J., Marsh, S., Peternelj, T., Briskey, D., et al. (2014). Altering the redox state of skeletal muscle by glutathione depletion increases the exercise-activation of PGC-1 $\alpha$. Physiol. Rep. 2:e12224. doi: 10.14814 /phy2.12224

Talbot, J., and Maves, L. (2016). Skeletal muscle fiber type: using insights from muscle developmental biology to dissect targets for susceptibility and resistance to muscle disease. Wiley Interdiscip. Rev. Dev. Biol. 5, 518-534. doi: 10.1002/wdev.230

Talha, S., Bouitbir, J., Charles, A. L., Zoll, J., Goette-Di Marco, P., Meziani, F., et al. (2013). Pretreatment with brain natriuretic peptide reduces skeletal muscle mitochondrial dysfunction and oxidative stress after ischemiareperfusion. J. Appl. Physiol. 114, 172-179. doi: 10.1152/japplphysiol.002 39.2012

Taylor, S. M., Cull, D. L., Kalbaugh, C. A., Senter, H. F., Langan, E. M. III., Carsten, C. G. III, et al. (2009). Comparison of interventional outcomes according to preoperative indication: a single center analysis of 2,240 limb revascularizations. J. Am. Coll. Surg. 208, 770-778. discussion: 778-780. doi: 10.1016/j.jamcollsurg.2009.01.025

Thaveau, F., Zoll, J., Rouyer, O., Chafke, N., Kretz, J. G., Piquard, F., et al. (2007). Ischemic preconditioning specifically restores complexes I and II activities of the mitochondrial respiratory chain in ischemic skeletal muscle. J. Vasc. Surg. 46, 541-547. discussion: 547. doi: 10.1016/j.jvs.2007.04.075

Tran, T. P., Tu, H., Pipinos, I. I., Muelleman, R. L., Albadawi, H., and Li, Y. L. (2011). Tourniquet-induced acute ischemia-reperfusion injury in mouse skeletal muscles: involvement of superoxide. Eur. J. Pharmacol. 650, 328-334. doi: 10.1016/j.ejphar.2010.10.037

Turóczi, Z., Arányi, P., Lukáts, A., Garbaisz, D., Lotz, G., Harsanyi, L., et al. (2014). Muscle fiber viability, a novel method for the fast detection of ischemic muscle injury in rats. PLOS ONE 9:e84783. doi: 10.1371/journal.pone.00 84783

Vignaud, A., Hourde, C., Medja, F., Agbulut, O., Butler-Browne, G., and Ferry, A. (2010). Impaired skeletal muscle repair after ischemia-reperfusion injury in mice. J. Biomed. Biotechnol. 2010:724914. doi: 10.1155/2010/724914

Woitaske, M. D., and McCarter, R. J. (1998). Effects of fiber type on ischemiareperfusion injury in mouse skeletal muscle. Plast. Reconstr. Surg. 102, 2052-2063. doi: 10.1097/00006534-199811000-00037

Yassin, M. M., Harkin, D. W., Barros D'Sa, A. A., Halliday, M. I., and Rowlands, B. J. (2002). Lower limb ischemia-reperfusion injury triggers a systemic inflammatory response and multiple organ dysfunction. World J. Surg. 26, 115-121. doi: 10.1007/s00268-001-0169-2

Yun, J., and Finkel, T. (2014). Mitohormesis. Cell Metab. 19, 757-766. doi: 10.1016/j.cmet.2014.01.011

Zhang, D., Wang, D., Pipinos, I. I., Muelleman, R. L., and Li, Y. L. (2017). Dexamethasone promotes long-term functional recovery of neuromuscular junction in a murine model of tourniquet-induced ischaemia-reperfusion. Acta Physiol. 219, 453-464. doi: 10.1111/apha.12737

Zoccarato, F., Cavallini, L., and Alexandre, A. (2004). Respiration-dependent removal of exogenous $\mathrm{H} 2 \mathrm{O} 2$ in brain mitochondria: inhibition by $\mathrm{Ca} 2+$. J. Biol. Chem. 279, 4166-4174. doi: 10.1074/jbc.M308143200

Zuo, L., and Pannell, B. (2015). Redox characterization of functioning skeletal muscle. Front. Physiol. 18, 6:338. doi: 10.3389/fphys.2015.00338

Zuo, L., Zhou, T., Pannell, B., Ziegler, A., and Best, T. (2015). Biological and physiological role of reactive oxygen species-the good, the bad and the ugly. Acta Physiol. 214, 329-348. doi: 10.1111/apha.12515

Conflict of Interest Statement: The authors declare that the research was conducted in the absence of any commercial or financial relationships that could be construed as a potential conflict of interest.

The reviewer ZL and handling Editor declared their shared affiliation, and the handling Editor states that the process nevertheless met the standards of a fair and objective review.

Copyright (c) 2017 Charles, Guilbert, Guillot, Talha, Lejay, Meyer, Kindo, Wolff, Bouitbir, Zoll and Geny. This is an open-access article distributed under the terms of the Creative Commons Attribution License (CC BY). The use, distribution or reproduction in other forums is permitted, provided the original author(s) or licensor are credited and that the original publication in this journal is cited, in accordance with accepted academic practice. No use, distribution or reproduction is permitted which does not comply with these terms. 